\title{
Distribution of Toenail Selenium Levels in Young Adult Caucasians and African Americans in the United States: The CARDIA Trace Element Study
}

\author{
Pengcheng Xun, MD, PhD ${ }^{1,2}$, Deborah Bujnowski, PhD, RD ${ }^{1,2}$, Kiang Liu, $\mathrm{PhD}^{3}$, J. Steve \\ Morris, $\mathrm{PhD}^{4}$, Zhongqin Guo, MD, MPH ${ }^{5,1}$, and Ka He, MD, ScD ${ }^{1,2}$ \\ ${ }^{1}$ Department of Nutrition, Gillings School of Global Public Health and School of Medicine, \\ University of North Carolina at Chapel Hill, NC, USA \\ 2 Department of Epidemiology, Gillings School of Global Public Health, University of North \\ Carolina at Chapel Hill, Chapel Hill, NC, USA \\ ${ }^{3}$ Department of Preventive Medicine, Feinberg School of Medicine, Northwestern University, \\ Chicago, IL, USA \\ ${ }^{4}$ Research Reactor Center, University of Missouri-Columbia, Columbia, MO, USA \\ ${ }^{5}$ Department of Epidemiology \& Biostatistics, School of Public Health, Ningxia Medical \\ University, Ningxia, P.R.China
}

\begin{abstract}
Background-Data on selenium (Se) levels in American young adults, especially in African Americans, are lacking.

Objective-This study presented toenail Se distributions in American young adults of both genders, including both Caucasians and African Americans; and explored potential predictors of toenail Se levels.

Data and Methods-Data from the Coronary Artery Risk Development in Young Adults study among 4252 American young adults, aged 20-32 in 1987 was used to examine toenail Se levels by instrumental neutron-activation analysis. The distribution of Se levels was described and multivariable linear regression was used to examine potential modifiers of toenail Se concentration within ethnicity-gender subgroups.
\end{abstract}

Results-The geometric mean of toenail Se in this cohort was $0.844 \mu \mathrm{g} / \mathrm{g}(95 \% \mathrm{CI}, 0.840-0.849$ $\mu \mathrm{g} / \mathrm{g}$ ) and the median was $0.837 \mu \mathrm{g} / \mathrm{g}(95 \% \mathrm{CI}, 0.833-0.844 \mu \mathrm{g} / \mathrm{g})$. Median levels from lowest to highest quintile were $0.691,0.774,0.838,0.913$ and $1.037 \mu \mathrm{g} / \mathrm{g}$, respectively. Se levels varied geographically, and were generally in accordance with its concentrations in local soil. Males, African Americans, current smokers, heavy drinkers and less educated participants were more likely to have low Se levels.

(C) 2011 Elsevier Inc. All rights reserved.

Corresponding author: Ka He, Departments of Nutrition and Epidemiology, Gillings School of Global Public Health and Department of Nutrition, School of Medicine, University of North Carolina at Chapel Hill, 2221 McGavran-Greenberg, Campus Box: 7461, Chapel Hill, NC, 27599, USA. Phone: 01-919-843-2476; Fax: 01-919-843-7216. kahe@ unc.edu..

Publisher's Disclaimer: This is a PDF file of an unedited manuscript that has been accepted for publication. As a service to our customers we are providing this early version of the manuscript. The manuscript will undergo copyediting, typesetting, and review of the resulting proof before it is published in its final citable form. Please note that during the production process errors may be discovered which could affect the content, and all legal disclaimers that apply to the journal pertain. 
Conclusion-This study suggests that toenail Se levels vary geographically depending on soil Se concentrations. In addition to gender, ethnicity and education level, smoking status and alcohol consumption are two important indicators of Se status since they are modifiable lifestyle factors. Findings from this study might aid public health professionals in identifying people at relatively high or low Se levels, so that chronic disease prevention efforts can be directed toward these subgroups.

\section{Keywords}

Selenium; toenail; distribution; American young adults; ethnicity

\section{Introduction}

Selenium (Se) presents a nutritional challenge because of its dual features as an essential trace element and a potential toxin. Severe deficiency of Se can lead to Keshan disease (a congestive cardiomyopathy)(Keshan Disease Research Group, 1979) and also contributes to Kashin-Beck disease (an endemic osteoarthropathy).(Diplock, 1987) Both were reported in areas of China and other countries until they were virtually eradicated with $\mathrm{Se}$ supplementation. The apparent benefits of Se may not be limited to alleviating overt deficiency. Low serum, plasma, erythrocyte and toenail Se statuses have been associated with increased risk of cardiovascular disease (CVD) and cancer in some epidemiological studies(Burguera et al., 1990; Flores-Mateo et al., 2006; Knekt et al., 1990; Knekt et al., 1998; Russo et al., 1997; van den Brandt et al., 2003; Willett et al., 1983; Yoshizawa et al., 1998; Young and Lee, 1999), illustrating Se's important role in human nutrition. Conversely, Se toxicity can occur in areas with elevated soil concentrations or from high-dose supplementation (Helzlsouer et al., 1985; Longnecker et al., 1991; MacFarquhar et al., 2010; Yang et al., 1989a; Yang et al., 1989b; Yang et al., 1983), though these scenarios are uncommon in the United States (US). Because deficient and excessive levels of Se can both be harmful to human health, the need for further study to assess individual Se intake and tissue levels is clear.

The primary dietary sources of Se are meat, poultry, fish, grains and cereals. Se levels in food are mainly determined by soil levels, which vary widely throughout the US.(Shacklette and Boerngen, 1984) Accurate and adequate assessment of Se intake based on individual food consumption is exceptionally difficult because of the minute amounts and wide variations in the same foods grown in different areas.(Willett, 1998) Also, estimating Se intake directly in meals is not feasible in large studies. Thus, assay of biological specimens, which reflects consumption, is preferred both as a measure of intake and as a means to validate other forms of exposure assessment.(Willett, 1998) One drawback of using blood (including whole blood, serum and plasma) or urine samples to identify Se status in the human body is that they respond to and reflect only recent changes. Even Se levels in erythrocytes may not reflect longer-term exposure to Se, as erythrocytes can only circulate for about 120 days in the body.(Joliet, 1953) Se measured in human toenails is more reliable than other body measures because: 1 ) toenails can reflect a time-integrated measure of exposure and have successfully predicted Se intake (R-square $=0.52$ ); (Longnecker et al., 1996) 2) they are less prone to superficial contamination than hair because of their lower surface: volume ratio; 3 ) they are environmentally sheltered in populations that wear shoes; (Hunter et al., 1990a) 4) they are easily collected, transported, stored and cleaned.(Morris et al., 1983) Toenail Se concentrations are highly correlated with Se levels in other critical organs; therefore they are very useful in large-scale epidemiological studies.(Morris, 2004) Thus, toenail Se measurements are being increasingly used in human studies. 
Previous studies have reported data on Se levels in the US and worldwide.(Hunter et al., 1990a; Kotsopoulos et al., 2010; Yoshizawa et al., 2003) However, these studies were conducted among middle-aged or older populations or only in one gender group. In particular, data on African Americans are limited. Therefore, we aim to present toenail Se distributions in American young adults of both genders, including both Caucasians and African Americans; and to explore potential predictors of toenail Se levels using data from the Coronary Artery Risk Development in Young Adults (CARDIA) Trace Element Study.

\section{Methods}

\subsection{Study population}

The CARDIA cohort was established in 1985 when 5115 American young adults, 18 to 30 years of age, participated in a study of the psychological and other lifestyle factors that might affect the evolution of coronary artery disease risk. Details of the study design have been published elsewhere.(Friedman et al., 1988) Briefly, the cohort was enrolled from four US cities including Birmingham, Alabama; Chicago, Illinois; Minneapolis, Minnesota; and Oakland, California and was roughly balanced by age (18-24 and 25-30 years), gender, ethnicity (African American and Caucasian), and education (high school or below and beyond high school). The CARDIA Trace Element Study was based on the CARDIA study and aimed to examine the potential association of certain trace elements such as selenium (Se), chromium $(\mathrm{Cr})$, mercury $(\mathrm{Hg})$, Arsenic $(\mathrm{As})$, Cadmium $(\mathrm{Cd})$ and Lead $(\mathrm{Pb})$ with CVD risk factors among American young adults.

In the present study, a total of 4624 participants attended the first follow-up examination in 1987, of which 4362 (94.3\%) participants provided toenail clippings.(Xun et al., 2010b) In this analysis, we excluded participants who had missing data on toenail weight $(n=75)$ and those with extremely high levels of toenail selenium $(\geq 2 \mu \mathrm{g} / \mathrm{g}, \mathrm{n}=28)$, as this high concentration is likely due to exogenous contamination based on our laboratory experience. (Morris et al., 2008) We also excluded those who had missing information on demographic data $(n=7)$. After these baseline exclusions, 4252 participants were included in this crosssectional analysis.

All participants signed an informed consent, and the study design, data collection and analyses were approved by the institutional review boards of the participating institutions.

\subsection{Toenail selenium measurements}

Toenail clippings were collected with a stainless steel clipper from all 10 toes by the participants themselves during the clinical examination. All toenail clippings were cleaned by sonication in $10 \%$ nitric acid followed by high-purity water using 10-minute exposure times. The nail clippings were then captured on a filter paper, rinsed thoroughly with highpurity water, and freeze dried. Selenium levels were analyzed by instrumental neutronactivation analysis (NAA) at the University of Missouri Research Reactor using a modification of the method previously described.(Morris et al., 1983) Toenail specimens were treated in random order by laboratory personnel who were blinded to other clinical measures. The average coefficient of variation for Se concentration in duplicate subsamples of toenails was $2.45 \%$ in the present study. In a pilot study conducted among 64 randomly selected CARDIA participants from the Chicago Field Center intended to examine withinperson variability of toenail Se measurements over time, the Spearman correlation between toenail selenium concentrations in toenail clippings collected 20 years apart and measured by the same laboratory using the same protocol was 0.56 .(Xun et al., 2010a) 


\subsection{Covariates}

Demographic variables were collected via questionnaire. Smoking status was determined according to self report and serum cotinine. Participants were classified into four groups: current smokers; former smokers; never smokers with passive exposure (self-reported never smokers with serum cotinine concentrations 1 to $15 \mathrm{ng} / \mathrm{ml}$ ); and never smokers without passive exposure (self-reported never smoker with non-detectable serum cotinine). Serum cotinine concentration over $15 \mathrm{ng} / \mathrm{ml}$ was used to determine current smokers who denied smoking.(Houston et al., 2006) Alcohol intake was assessed through a self-administered questionnaire and interview-based dietary history. Education levels were classified into two groups, $\leq 16$ vs. $>16$ years of education.

\subsection{Statistical methods}

The distribution of toenail Se levels was described through the calculation of percentiles and geometric means. Natural logarithm transformation was used to improve the normality of the Se distribution. Exact 95\% confidence intervals (CIs) for percentiles of Se were calculated using a binomial-based method,(StataCorp LP., 2009) while 95\% CIs for geometric means were calculated by exponentiating the confidence limits of the arithmetic mean of $\ln (\mathrm{Se})$. Analysis of variance was used to compare the geometric mean with the reference by using $\ln (\mathrm{Se})$ as the dependent variable; this was followed by Scheffe test for multiple comparisons as appropriate. Kernel density estimation with a Parzen function was used to graph ethnicity-specific distributions of toenail Se concentrations within each gender group.(Vogt et al., 2007)

Using multivariate linear regression, $\ln (\mathrm{Se})$ was regressed against age, gender, ethnicity, study center, smoking status, alcohol consumption and education level as independent variables to examine whether these variables were independently associated with toenail $\mathrm{Se}$ levels. Wald $\mathrm{Z}$ statistics were calculated to test the null hypotheses that the beta coefficients were not different from zero.

All analyses were performed using STATA 11.0 (Stata Corporation LP, College Station, Texas, US). $\mathrm{P} \leq 0.05$ was considered statistically significant.

\section{Results}

Geometric means and selected percentiles with 95\% CIs for toenail Se levels, overall and from subgroups defined by selected variables, are shown in Table 1 . The overall geometric mean concentration of toenail Se was $0.844 \mu \mathrm{g} / \mathrm{g}(95 \% \mathrm{CI}, 0.840-0.849 \mu \mathrm{g} / \mathrm{g})$. Median levels from lowest to highest quintile were $0.691,0.774,0.838,0.913$ and $1.037 \mu \mathrm{g} / \mathrm{g}$. Average toenail Se levels from Birmingham were the lowest, while those from Minneapolis were the highest. Women, Caucasians, non-current smokers and those with a college education or beyond had higher Se levels compared to men, African Americans, current smokers and those with less than a college education, respectively. Participants in the highest category of alcohol consumption ( $\geq 24 \mathrm{ml} / \mathrm{d}$ ) had lower Se levels than those with medium consumption $(12-23.9 \mathrm{ml} / \mathrm{d})$, and even lower than those with low or no alcohol consumption $(<12 \mathrm{ml} / \mathrm{d})$.

Density estimates (Figure 1 and 2) illustrate that, for both genders, toenail Se concentrations were shifted noticeably lower in African Americans relative to Caucasians. This pattern was observed across all four study centers (figure not shown)

Results of multivariate linear regression analyses are shown in Table 2. $\mathrm{Ln}(\mathrm{Se})$ concentrations served as the dependent variables and were modeled for each of the four gender-ethnicity subgroups. Age was significantly and inversely associated with Se levels only in African American women. For every 5 year increment in age, toenail $\ln (\mathrm{Se})$ was 
$0.01 \mu \mathrm{g} / \mathrm{g}$ lower. The multivariable adjusted mean values of Se were, across study centers, highest to lowest in Minneapolis, Oakland, Chicago, and Birmingham, respectively for all gender-ethnicity subgroups. Current smokers had lower Se levels compared with noncurrent smokers in all gender-ethnicity subgroups. Alcohol consumption was independently and inversely associated with toenail Se levels in men but not in women. Education level was positively (borderline significant) associated with Se concentration only in African American men. The proportion of variation in Se levels explained by four ethnicity-gender specific regression models was low (R square $=23 \%, 17 \%, 18 \%$ and $15 \%$, respectively, in African American men, African American women, Caucasian men and Caucasian women).

\section{Discussion}

These results extend recently released estimates of toenail Se levels in American young adults including African Americans and Caucasians using data from the CARDIA Trace Element Study.(Xun et al., 2010b) In this study, toenail Se levels were associated with gender, ethnicity, study center, smoking status and alcohol consumption. Men, African Americans, those in Birmingham, current smokers, and heavy drinkers were more likely to have low Se levels. In African American women, those who were relatively older were more likely to have low Se levels, while Se levels were lower in African American men with less education.

There is substantial evidence that Se measurement in toenails is an excellent indicator of long-term selenium intake (Hunter et al., 1990b; Willett, 1987) and has been used widely in clinical and epidemiological studies.(Guallar et al., 2002; Yoshizawa et al., 2002) Of note, this cohort included approximately equal percentages of African Americans (48.5\%) and Caucasians, and men (45.3\%) and women, which makes it unique. In contrast, other studies with available toenail data such as the Nurses' Health Study and the Health Professionals Follow-Up Study have only reported Se levels in small scale case-control studies (Michaud et al., 2005; Yoshizawa et al., 2003), and these studies do not include toenail Se data on both genders and/or on African Americans. Importantly, as Se levels may change over time, our findings will provide valuable information on Se status 20 years ago, which may serve as a baseline or a reference for future studies, and also allow for testing trends or changes in $\mathrm{Se}$ levels in relation to chronic disease development.

A few limitations of this study should be considered when interpreting the findings. First, toenail Se provides a long-term estimate of Se itself, but is not a real-time marker of Se status, as are glutathione peroxidase (GSH-Px) and selenoprotein P. However, toenail Se levels are highly correlated with Se levels in whole blood and other organs and with dietary intake, all of which are also highly correlated with functional markers such as selenoprotein P.(Hurst et al., 2010) Second, CARDIA does not include a nationally representative sample, though sample selection was roughly balanced by gender and ethnicity, and every attempt was made to select a representative sample of young American adults from most of the selected metropolitan areas. Se status of CARDIA participants may be different from the general US population, and thus the generalizability of our findings may be limited.

This study showed that the median toenail Se level in CARDIA participants was $0.844 \mu \mathrm{g} / \mathrm{g}$. This was slightly higher than the median of $0.82 \mu \mathrm{g} / \mathrm{g}$ in 181 healthy control subjects in a case-control sub-study based on the Health Professionals Follow-up Study.(Yoshizawa et al., 1998) Our study also documented a wide range in levels, from a median of $0.691 \mu \mathrm{g} / \mathrm{g}$ in the bottom quintile to $1.037 \mu \mathrm{g} / \mathrm{g}$ in the top quintile. The difference in these two extreme quintiles is comparable to the range found in a sample of people living in the Boston area $(0.74 \mu \mathrm{g} / \mathrm{g})$, a region with a relatively low soil Se concentration, and another sample of people living in South Dakota $(1.17 \mu \mathrm{g} / \mathrm{g})$, an area with very high soil Se.(Morris et al., 1983) 
The aforementioned studies all detected toenail Se levels with the same method in the same lab as our study; conversely, it is difficult to compare our results with Se levels reported in some other studies, such as NHANES, which were detected in different biological samples (i.e., serum) with different analytic methods. Further studies focusing on toenail Se levels in the general population are warranted.

In the present study, toenail Se levels varied geographically. Average toenail Se levels in these four cities were generally in accordance with regional distribution of Se in soil as indicated by the U. S. Geological Survey (Shacklette and Boerngen, 1984), forage and grains (White and Zasoski, 1999); this suggests that regional variations in toenail Se levels directly reflect changes in dietary Se intake by region, then variations in forage and grains, and ultimately, the concentration of Se in soil within the US. Geographic differences may also be explained by dietary factors that might limit food consumption from the national food distribution system.(Monsen, 2000)

Although age has been reported to influence Se levels, our data fail to support any agerelated pattern except in African American women, who had a net decrease of $0.01 \mu \mathrm{g} / \mathrm{g}$ in $\ln (\mathrm{Se})$ per 5 year increment in age. Our finding for this subgroup might be explained by the general fact that Se intake and absorption may decrease, and excretion increase, with increasing age.(Lloyd et al., 1983) The gender heterogeneity observed for Se levels may be due to differences in Se intake and bioavailability between males and females. Although men generally consume more food than women and have higher Se intake as a result, their Se levels did not reflect greater exposure in this study sample; consistent with results from previous studies(Swanson et al., 1990), this study documented higher Se levels among women than men. No evidence has been found to support that men absorb less Se than women. Higher Se levels in women relative to men might be partially explained by higher estrogen levels. A cross-sectional study of three generations (daughters, mothers and grandmothers) with mean age of 24,50 and 75 , respectively, reported that serum Se concentrations of mothers $(135 \mathrm{ng} / \mathrm{mL})$ were significantly greater than those of daughters $(116 \mathrm{ng} / \mathrm{mL})$ and grandmothers $(110 \mathrm{ng} / \mathrm{mL})$, which indicated that Se status fluctuated during the female life cycle and was related to estrogen status.(Smith et al., 2000)

Our data indicate an obvious racial difference in density of toenail Se within each gender subgroup. Racial variation in Se status has been reported previously.(Glauser et al., 1999; Goodman et al., 2001; Niskar et al., 2003; Vogt et al., 2003; Vogt et al., 2007; Willett et al., 1983) For example, the data from third National Health and Nutrition Examination Survey (NHANES) indicated that the Blacks had lower serum selenium concentrations relative to Whites.(Vogt et al., 2007) However, most of these studies have been limited by the inclusion of relatively few African Americans. Although the mechanism for racial variation observed in this study is unclear, there are several potential explanations for our findings. First, Se intake was slightly higher among Caucasians than African Americans (Vogt et al., 2007) and differential intake of Se-rich foods may play an important role in determining Se status. Second, genetic variation between ethnicities could affect the absorption, metabolism and excretion of Se and thus Se concentrations in the body.(Hu et al., 2001; Rayman, 2005) Third, although we adjusted for known potential confounders and included certain predictors in the model, we cannot rule out the possibility of unknown confounders explaining the variation observed.

In this study, smoking status remained an independent indicator of toenail Se levels across all ethnicity-gender subgroups. A European study documented that persons who currently smoked cigarettes had significantly lower toenail Se levels than ex-smokers and never smokers.(Kardinaal et al., 1997) Similarly, toenail Se levels were found to be lower in smokers than non-smokers in other studies.(Ghadirian et al., 2000; Krogh et al., 2003; 
Swanson et al., 1990) Although the dietary habits of smokers have not been adequately studied, there is published evidence that lower levels of Se can be attributed either to the nature of tobacco, which reduces Se absorption, or to smokers' reduced food intakes (Swanson et al., 1990) or their selection of foods containing less Se.(Fehily et al., 1984; Ghadirian et al., 2000) Toenail Se levels were lower in African American men who consumed $>12 \mathrm{ml} / \mathrm{d}$ of alcohol and in Caucasian men consuming $>24 \mathrm{ml} / \mathrm{d}$ compared to nondrinkers. This finding is supported by other studies (Dhindsa et al., 1998; Luty-Frackiewicz et al., 2002), but potential mechanisms are unclear. Decreased Se intake among those consuming more alcohol and reduced hepatic storage resulting from alcohol consumption might be involved.(Dutta et al., 1983)

In conclusion, using data from the CARDIA Trace Element Study, we observed that gender, ethnicity, study center, smoking status and alcohol consumption all influence toenail Se levels. Among these, alcohol consumption and smoking status are the most important indicators since they are two lifestyle habits for which we can promote some changes, e.g. cessation of smoking or control of alcohol use. Findings from this study might aid public health professionals in identifying people with relatively high or low Se levels, so that chronic disease prevention efforts can be directed toward these subgroups.

\section{Acknowledgments}

Funding Sources:

This study was supported by grants R01HL081572 and P30ES10126, and contracts N01-HC-48047, N01HC-48048, N01-HC-48049, N01-HC-48050, and N01-HC-95095 from the National Institutes of Health. The authors thank Dr. Jared Reis for his valuable comments and Wang Xin for verifying the STATA programming. The authors also thank the other investigators and staff of the Coronary Artery Risk Development in Young Adults (CARDIA) Study for their valuable contributions.

\section{References}

Burguera JL, et al. Blood serum selenium in the province of Merida, Venezuela, related to sex, cancer incidence and soil selenium content. J Trace Elem Electrolytes Health Dis. 1990; 4:73-7. [PubMed: 2136228]

Dhindsa HS, et al. Plasma selenium concentrations in a Sikh population in Sydney, Australia. Analyst. 1998; 123:885-7. [PubMed: 9709481]

Diplock AT. Trace elements in human health with special reference to selenium. Am J Clin Nutr. 1987; 45:1313-22. [PubMed: 3578121]

Dutta SK, et al. Selenium and acute alcoholism. Am J Clin Nutr. 1983; 38:713-8. [PubMed: 6637863]

Fehily AM, et al. Diet, smoking, social class, and body mass index in the Caerphilly Heart Disease Study. Am J Clin Nutr. 1984; 40:827-33. [PubMed: 6486090]

Flores-Mateo G, et al. Selenium and coronary heart disease: a meta-analysis. Am J Clin Nutr. 2006; 84:762-73. [PubMed: 17023702]

Friedman GD, et al. CARDIA: study design, recruitment, and some characteristics of the examined subjects. J Clin Epidemiol. 1988; 41:1105-16. [PubMed: 3204420]

Ghadirian P, et al. A case-control study of toenail selenium and cancer of the breast, colon, and prostate. Cancer Detect Prev. 2000; 24:305-13. [PubMed: 11059562]

Glauser TA, et al. Racial differences in free radical scavenging enzyme activity in children. J Child Neurol. 1999; 14:382-7. [PubMed: 10385846]

Goodman GE, et al. Predictors of serum selenium in cigarette smokers and the lack of association with lung and prostate cancer risk. Cancer Epidemiol Biomarkers Prev. 2001; 10:1069-76. [PubMed: 11588133]

Guallar E, et al. Mercury, fish oils, and the risk of myocardial infarction. N Engl J Med. 2002; 347:1747-54. [PubMed: 12456850] 
Helzlsouer K, et al. Acute selenium intoxication in the United States. Federation Proceedings. 1985; 44:1670.

Houston TK, et al. Active and passive smoking and development of glucose intolerance among young adults in a prospective cohort: CARDIA study. BMJ. 2006; 332:1064-9. [PubMed: 16603565]

$\mathrm{Hu}$ YJ, et al. Distribution and functional consequences of nucleotide polymorphisms in the 3'untranslated region of the human Sep15 gene. Cancer Res. 2001; 61:2307-10. [PubMed: 11280803]

Hunter DJ, et al. Predictors of selenium concentration in human toenails. Am J Epidemiol. 1990a; 132:114-22. [PubMed: 2356804]

Hunter DJ, et al. A prospective study of selenium status and breast cancer risk. JAMA. 1990b; 264:1128-31. [PubMed: 2384937]

Hurst R, et al. Establishing optimal selenium status: results of a randomized, double-blind, placebocontrolled trial. Am J Clin Nutr. 2010; 91:923-31. [PubMed: 20181815]

Joliet JF. Duration of the life span of erythrocytes. C R Hebd Seances Acad Sci. 1953; 237:1797-9.

Kardinaal AF, et al. Association between toenail selenium and risk of acute myocardial infarction in European men. The EURAMIC Study. European Antioxidant Myocardial Infarction and Breast Cancer. Am J Epidemiol. 1997; 145:373-9. [PubMed: 9054242]

Knekt $\mathrm{P}$, et al. Serum selenium and subsequent risk of cancer among Finnish men and women. J Natl Cancer Inst. 1990; 82:864-8. [PubMed: 2332904]

Knekt P, et al. Is low selenium status a risk factor for lung cancer? Am J Epidemiol. 1998; 148:97582. [PubMed: 9829869]

Kotsopoulos J, et al. Toenail selenium status and DNA repair capacity among female BRCA1 mutation carriers. Cancer Causes Control. 2010; 21:679-87. [PubMed: 20063075]

Krogh V, et al. Toenail selenium as biomarker: reproducibility over a one-year period and factors influencing reproducibility. J Trace Elem Med Biol. 2003; 17(Suppl 1):31-6. [PubMed: 14650626]

Lloyd B, et al. Effect of smoking, alcohol, and other factors on the selenium status of a healthy population. J Epidemiol Community Health. 1983; 37:213-7. [PubMed: 6619720]

Longnecker MP, et al. Use of selenium concentration in whole blood, serum, toenails, or urine as a surrogate measure of selenium intake. Epidemiology. 1996; 7:384-90. [PubMed: 8793364]

Longnecker MP, et al. Selenium in diet, blood, and toenails in relation to human health in a seleniferous area. Am J Clin Nutr. 1991; 53:1288-94. [PubMed: 2021136]

Luty-Frackiewicz A, et al. Effect of smoking and alcohol consumption on the serum selenium level of Lower Silesian population. Sci Total Environ. 2002; 285:89-95. [PubMed: 11874051]

MacFarquhar JK, et al. Acute selenium toxicity associated with a dietary supplement. Archives of internal medicine. 2010; 170:256-61. [PubMed: 20142570]

Michaud DS, et al. Toenail selenium concentrations and bladder cancer risk in women and men. Br J Cancer. 2005; 93:804-6. [PubMed: 16175184]

Monsen ER. Dietary reference intakes for the antioxidant nutrients: vitamin C, vitamin E, selenium, and carotenoids. J Am Diet Assoc. 2000; 100:637-40. [PubMed: 10863565]

Morris JS, et al. Quality control in the neutron activation analysis of biological markers for selenium in epidemiological investigations. Journal of Radioanalytical and Nuclear Chemistry. 2008; $276: 7-$ 13.

Morris JS, et al. Dietary selenium in humans: Toenails as an indicator. Biol Trace Elem Res. 1983; 5:529-37.

Morris, JS.; Veatch, AE.; Spate, VL. INAA deternibation of selenium distribution in castrated and intact dogs; 11th International Conference on Modern Trends in Activation Analysis; Guildford, UK: Viridian Publishing, University of Surrey; 2004. p. 161

Niskar AS, et al. Serum selenium levels in the US population: Third National Health and Nutrition Examination Survey, 1988-1994. Biol Trace Elem Res. 2003; 91:1-10. [PubMed: 12713024]

Rayman MP. Selenium in cancer prevention: a review of the evidence and mechanism of action. Proc Nutr Soc. 2005; 64:527-42. [PubMed: 16313696] 
Russo MW, et al. Plasma selenium levels and the risk of colorectal adenomas. Nutr Cancer. 1997; 28:125-9. [PubMed: 9290116]

Shacklette, HT.; Boerngen, JG. U.S. Dept. of the Interior For sale by the Distribution Branch. U. S.Geological Survey; Alexandria, VA: 1984. Element concentrations in soils and other surficial materials of the conterminous United States.

Smith AM, et al. Generational differences in selenium status of women. Biol Trace Elem Res. 2000; 75:157-65. [PubMed: 11051605]

StataCorp LP.. Getting started with Stata for Windows, release 11. StataCorp LP; College Station, Tex: 2009.

Swanson CA, et al. Selenium intake, age, gender, and smoking in relation to indices of selenium status of adults residing in a seleniferous area. Am J Clin Nutr. 1990; 52:858-62. [PubMed: 2239761]

van den Brandt PA, et al. Toenail selenium levels and the subsequent risk of prostate cancer: a prospective cohort study. Cancer Epidemiol Biomarkers Prev. 2003; 12:866-71. [PubMed: 14504196]

Vogt TM, et al. Serum selenium and risk of prostate cancer in U.S. blacks and whites. Int J Cancer. 2003; 103:664-70. [PubMed: 12494476]

Vogt TM, et al. Racial differences in serum selenium concentration: analysis of US population data from the Third National Health and Nutrition Examination Survey. Am J Epidemiol. 2007; 166:280-8. [PubMed: 17557900]

White JG, Zasoski RJ. Mapping soil micronutrients. Field Crops Research. 1999; 60:11-26.

Willett W. Nutritional epidemiology: issues and challenges. Int J Epidemiol. 1987; 16:312-7. [PubMed: 3610460]

Willett, WC. Nutritional Epidemiology. Oxford University Press; New York, NY: 1998.

Willett WC, et al. Prediagnostic serum selenium and risk of cancer. Lancet. 1983; 2:130-4. [PubMed: 6134981]

Xun P, et al. Longitudinal association between toenail selenium levels and measures of subclinical atherosclerosis: the CARDIA trace element study. Atherosclerosis. 2010a; 210:662-7. [PubMed: 20138620]

Xun P, et al. Associations of toenail selenium levels with inflammatory biomarkers of fibrinogen, high-sensitivity c-reactive protein, and interleukin-6: The CARDIA Trace Element Study. Am J Epidemiol. 2010b; 171:793-800. [PubMed: 20219762]

Yang G, et al. Studies of safe maximal daily dietary Se-intake in a seleniferous area in China. Part II: Relation between Se-intake and the manifestation of clinical signs and certain biochemical alterations in blood and urine. Journal of trace elements and electrolytes in health and disease. 1989a; 3:123-30. [PubMed: 2535331]

Yang G, et al. Studies of safe maximal daily dietary selenium intake in a seleniferous area in China. I. Selenium intake and tissue selenium levels of the inhabitants. J Trace Elem Electrolytes Health Dis. 1989b; 3:77-87. [PubMed: 2535327]

Yang GQ, et al. Endemic selenium intoxication of humans in China. Am J Clin Nutr. 1983; 37:87281. [PubMed: 6846228]

Yoshizawa K, et al. Prospective study of selenium levels in toenails and risk of coronary heart disease in men. Am J Epidemiol. 2003; 158:852-60. [PubMed: 14585763]

Yoshizawa K, et al. Mercury and the risk of coronary heart disease in men. N Engl J Med. 2002; 347:1755-60. [PubMed: 12456851]

Yoshizawa K, et al. Study of prediagnostic selenium level in toenails and the risk of advanced prostate cancer. J Natl Cancer Inst. 1998; 90:1219-24. [PubMed: 9719083]

Young KJ, Lee PN. Intervention studies on cancer. Eur J Cancer Prev. 1999; 8:91-103. [PubMed: 10335455] 


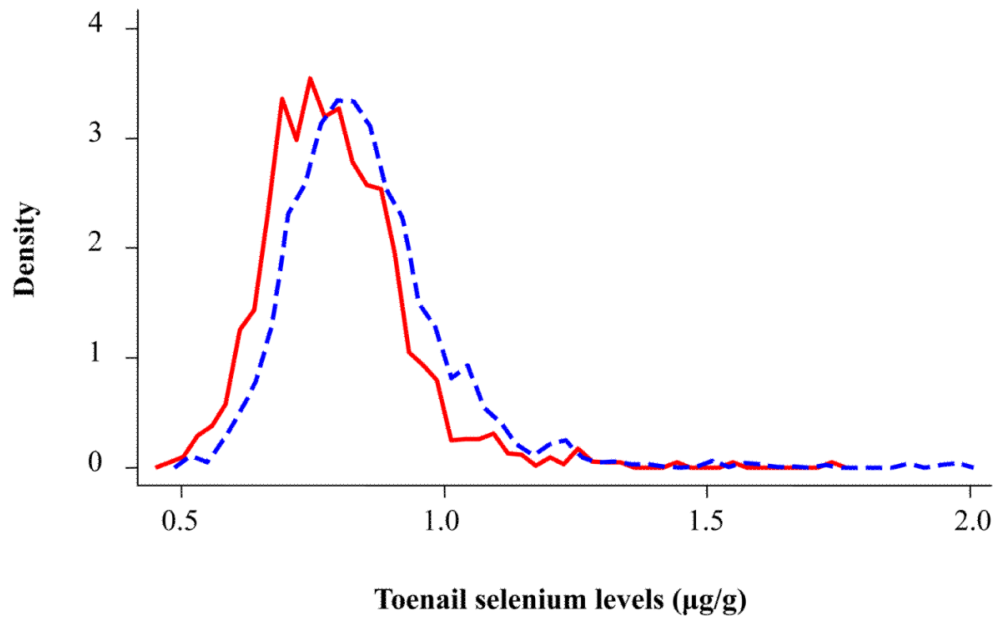

Figure 1.

Population kernel density estimates of toenail selenium concentration $(\mu \mathrm{g} / \mathrm{g})$ for African American and Caucasian men in CARDIA Trace Element Study, 1987. The solid curve represents the density for African Americans and the dash-line curve represents the density for Caucasians. The area under each curve above an interval represents the estimated population relative frequency. A Parzen kernel function was used for density estimates 


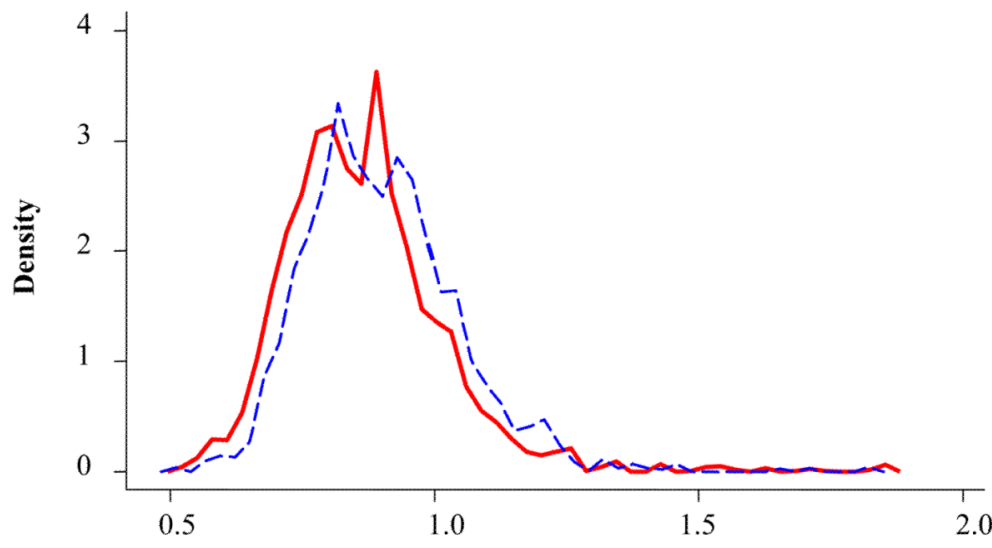

Toenail selenium levels $(\mu \mathrm{g} / \mathrm{g})$

Figure 2.

Population kernel density estimates of toenail selenium concentration $(\mu \mathrm{g} / \mathrm{g})$ for African American and Caucasian women in CARDIA Trace Element Study, 1987. The solid curve represents the density for African Americans and the dash-line curve represents the density for Caucasians. The area under each curve above an interval represents the estimated population relative frequency. A Parzen kernel function was used for density estimates. 


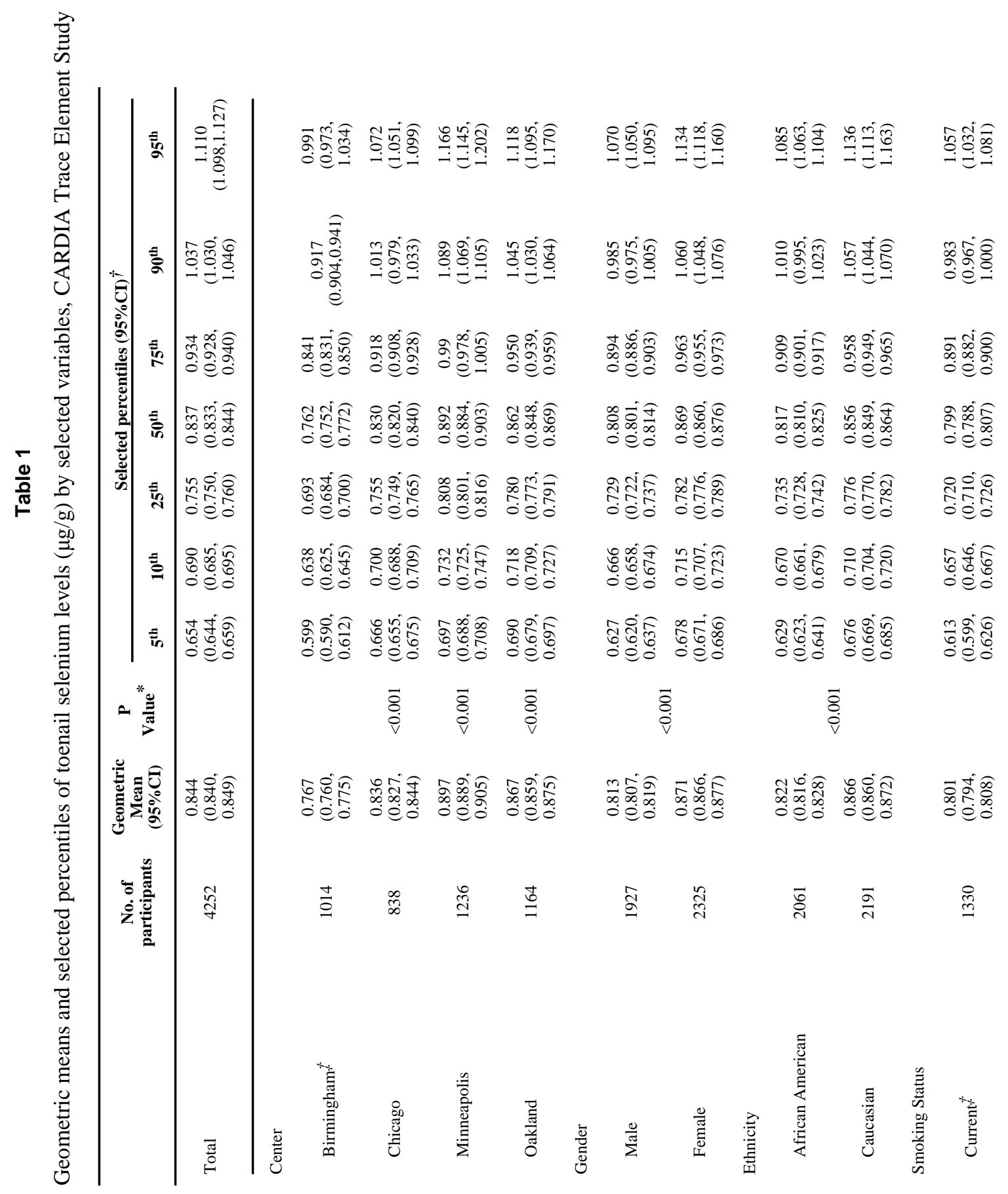




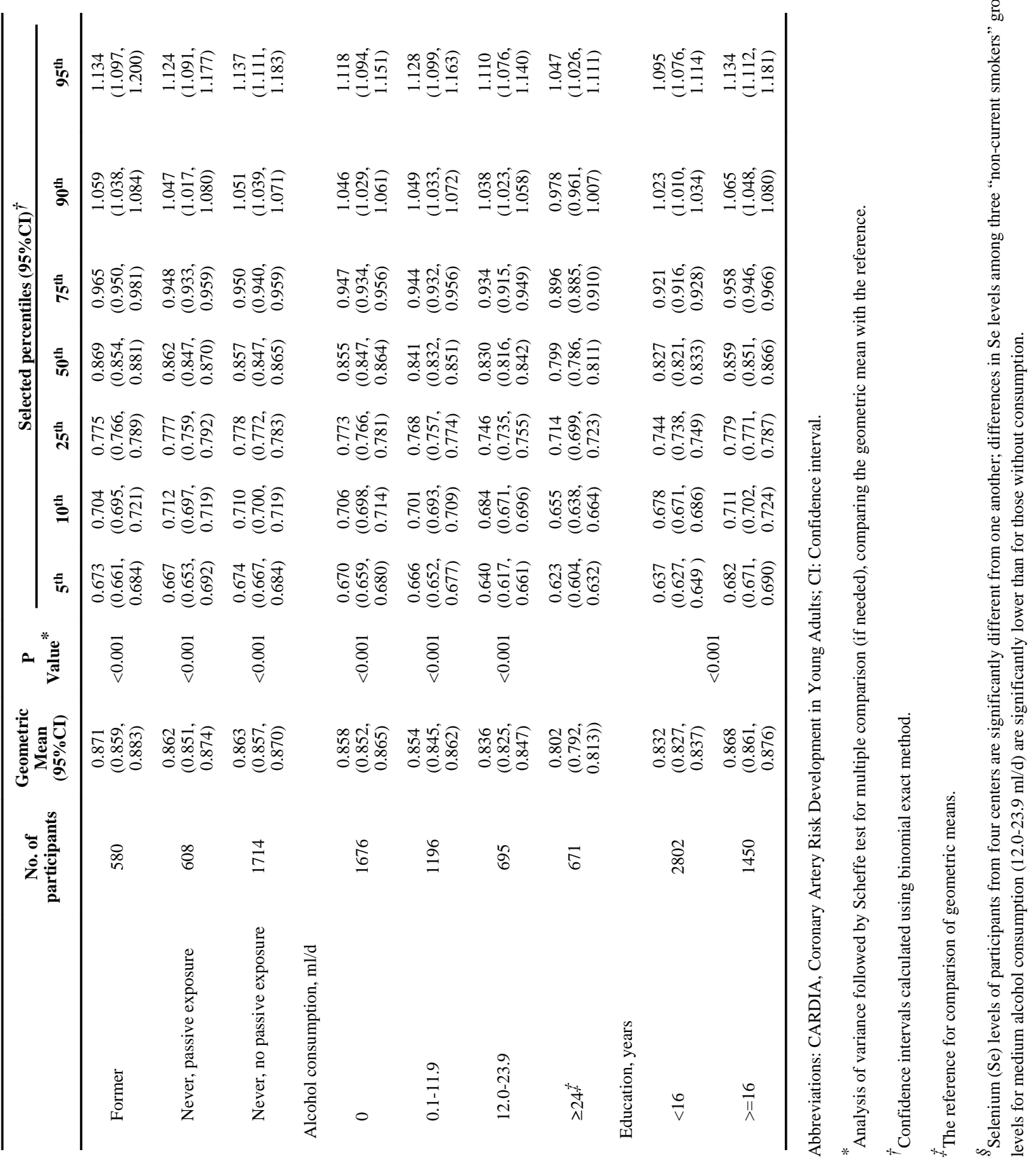




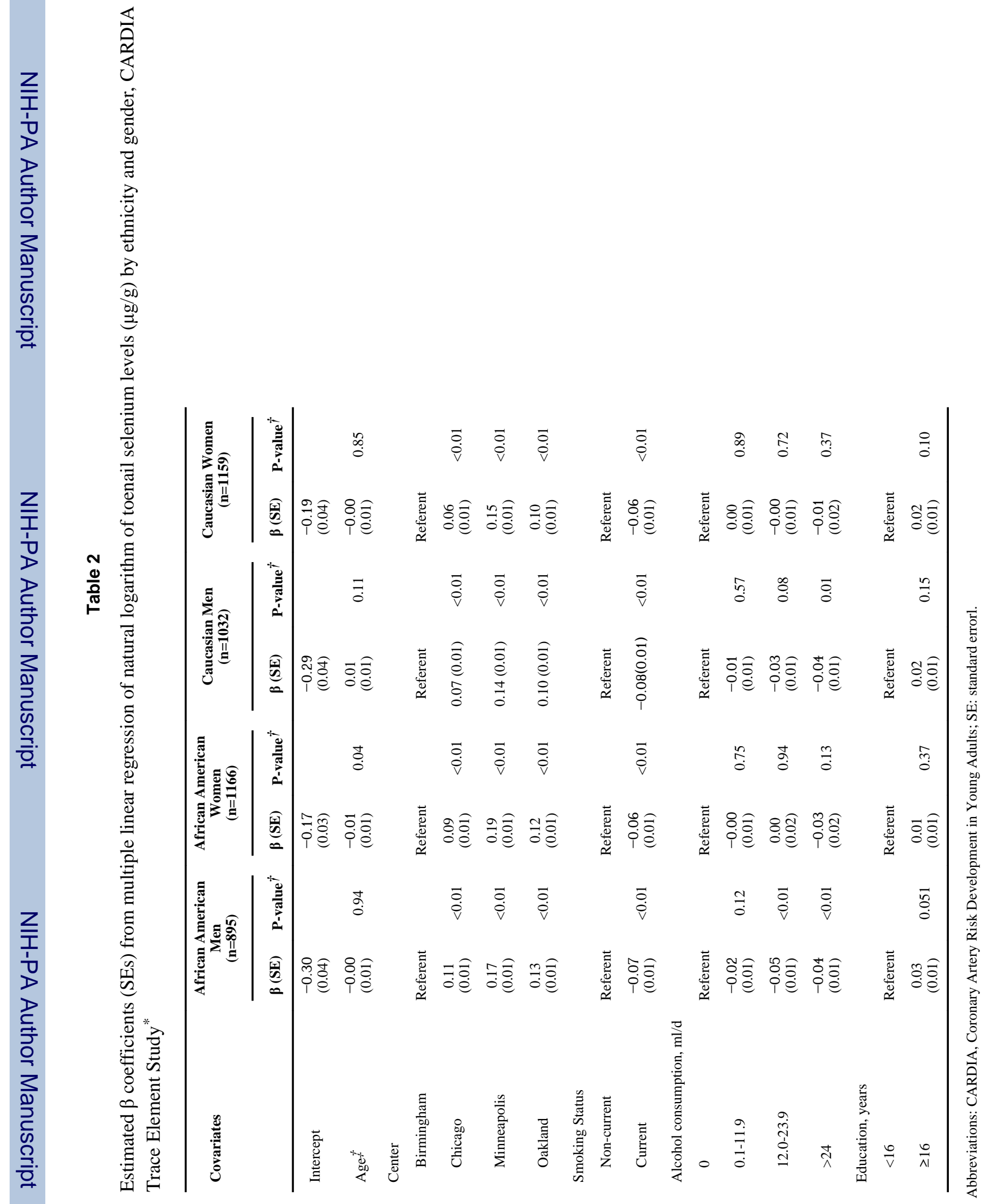




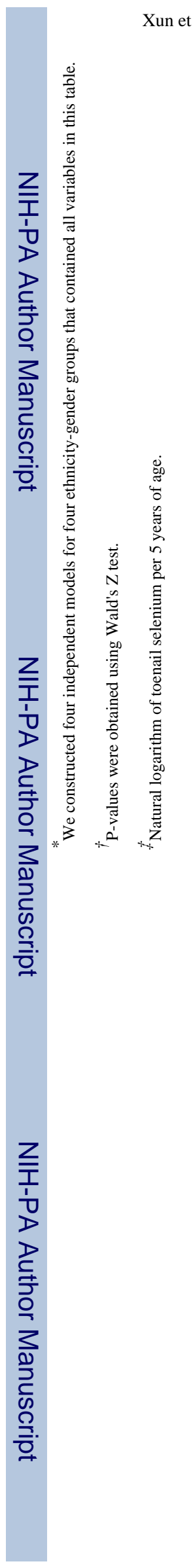

Environ Res. Author manuscript; available in PMC 2012 May 1. 